\title{
VERSATILE VACUUM PACKAGING FOR EXPERIMENTAL STUDY OF RESONANT MEMS
}

\author{
Adam R. Schofield, Alexander A. Trusov, and Andrei M. Shkel \\ University of California, Irvine, USA
}

\begin{abstract}
This paper presents a versatile sub-mTorr vacuum packaging approach ideally suited for R\&D of high performance dynamic MEMS. The procedure takes advantage of processing steps and materials used throughout the MEMS industry while providing maximum flexibility for changes in die size and layout. Prototypes of a new tuning fork gyroscope concept optimized to minimize substrate energy dissipation were packaged using the presented techniques and experimentally characterized to determine the sealed vacuum level. The combination of high-Q mechanical design with the packaging process resulted in a stable quality factor of greater than 91,000 .
\end{abstract}

\section{INTRODUCTION}

As micromachined devices become more sophisticated and complex, the role of packaging becomes increasingly important. The package is responsible for protecting the fragile mechanical parts from the environment while also providing electrical connections to the macro world. In particular, resonant MEMS are often sealed at vacuum in order to minimize energy loss and improve signal-to-noise ratio. The fact that packaging typically accounts for a majority of the overall cost further underscores its importance in achieving and maintaining optimum device operation $[1,2]$.

A significant amount of both academic and industrial research has been focused on developing alternative packaging solutions aimed at maximizing the performance of micromachined sensors [2, 3]. Since a majority of these packaging approaches are ultimately targeted for commercialization, they generally rely on the hermetic encapsulation of the mechanical sensor element on a wafer-scale. While wafer-level packaging is the preferable approach for mature, high-volume applications due its potential for size miniaturization, it is not well suited for research and development of MEMS prototypes where multiple concepts are often explored in parallel and iterated several times.

This work presents a detailed description of a flexible methodology using a package-level vacuum sealing technique that can be applied to a broad range of substrate materials, device layouts, and die sizes. The approach relies on a combination of standard tech- niques and materials used throughout the MEMS industry about which little is reported in literature. This includes two separate, sequential steps: the fluxless, eutectic attachment of a MEMS die to a package followed by the hermetic sealing of the device within the cavity at vacuum, maintained and stabilized using getter material.

When these packaging techniques are combined with mechanically optimized resonant MEMS structures, such as the new high-Q, dual mass gyroscope described in [4], vacuum sealed prototypes with standalone quality factors approaching 100,000 can be obtained. The technology is also equally suited for applications requiring the low volume production of ultra high performance sensors where package miniaturization is not the highest priority.

\section{PACKAGING PROCEDURE}

The following sections present the complete process details for the versatile packaging procedure developed for achieving sub-mTorr cavity pressures. For this work, a 24 pin ceramic dual-inline package (DIP) from Spectrum Semiconductor Materials (part number CSB02491) was used for the vacuum sealing.

\section{Bake Out}

The first, sometimes overlooked step toward achieving a successful vacuum packaging run is the bake out. After hermetically sealing a cavity, an observed degradation of pressure is generally observed due to the desorption of trapped gases from the package; when nickel and gold are electroplated, for example, a significant amount of hydrogen can become trapped in the metals making the package itself a major source of outgassing [5]. A proper bake out consists of holding the package at an elevated temperature in an ultra-high vacuum environment $\left(<10^{-4}\right.$ Torr $)$ for an extended amount of time. If the packages are held at too high of a temperature for too long, nickel can diffuse into the gold thereby limiting or possibly eliminating the ability to die attach and wire-bond. It is suggested that a temperature of $400{ }^{\circ} \mathrm{C}$ for no longer than 1 hour can effectively drive out a majority of the trapped gas while also limiting nickel diffusion [5]. Any bake out, even a more conservative one of $250-300{ }^{\circ} \mathrm{C}$ for duration of 3 to 72 hours, will minimize outgassing and help maintain the vacuum level in the sealed cavity. 


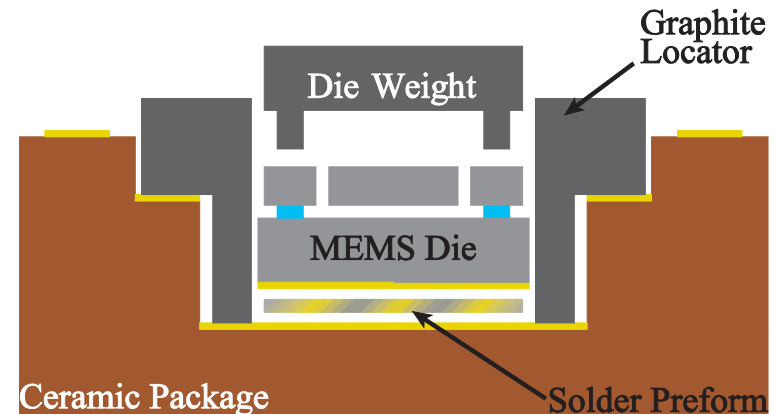

Figure 1. Schematic depicting the package level, fluxless die attachment process using 80/20 gold-tin eutectic solder.

\section{Die Attachment}

The next step in the versatile vacuum packaging procedure is the attachment of the MEMS die to the package. A schematic is presented in Figure 1 that depicts the components used during this process. It begins with the insertion of a graphite locator into the gold plated cavity of an appropriately sized package. Next, a solder preform is placed in the bottom of cavity through the opening in the locator frame followed by the device to be attached. The MEMS die must have a backside metallization layer to ensure that the solder will wet during the attachment process; for this work, a $500 \AA$ chromium adhesion layer followed by $5000 \AA$ of gold was used. The locator aids in the placement of the solder preform and the MEMS die as it centers the components within the cavity and limits their motion during the subsequent reflow processes.

The bonding material chosen for the die attachment process is the well known eutectic solder alloy comprised of 20 weight percent tin with 80 weight percent gold (80/20 Au-Sn). One of the motivating factors for using 80/20 Au-Sn solder is the extremely strong bond that is formed between the gold coated surfaces of the die and package. Since no flux or epoxy is used, it produces little outgassing making it compatible with the subsequent high vacuum sealing step. Additionally, the rigid attachment provided by the 80/20 goldtin solder also allows the highest possible limiting quality factor for 1-DOF and unbalanced 2-DOF resonant systems operated in vacuum, thereby helping to maximize the signal-to-noise ratio of the sensor [6].

While the gold-tin solder has good wettability, its high surface tension means that pressure must be applied to obtain a void-free bond [7]. This poses a problem as released MEMS devices typically have fragile mobile parts which can be easily broken. Therefore, a precision graphite die weight was manufactured to safely apply force of up to approximately $1 \mathrm{~N}$ to the die on the anchored outer frame. It is aligned to the device using the locator and if necessary, serves as a platform for any additional force to be applied during the re-

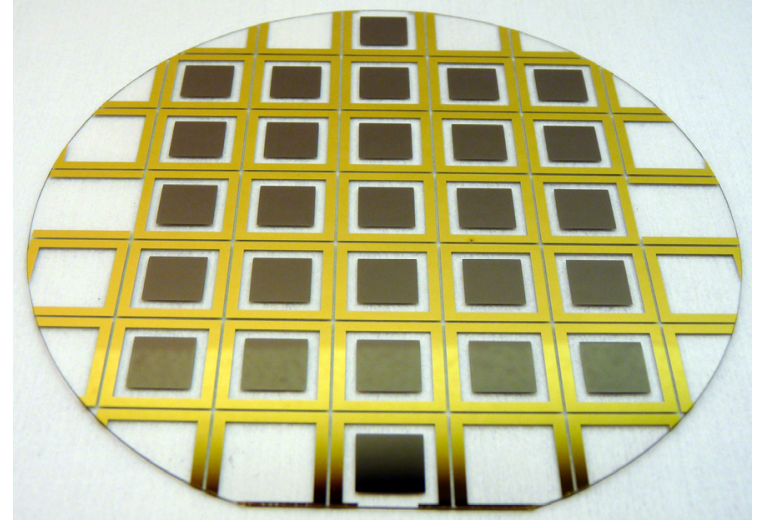

Figure 2. Photograph of custom glass lid wafer with getter material for ultra-high vacuum sealing.

flow. Once in place, the system is heated above the gold-tin eutectic melting temperature of $278{ }^{\circ} \mathrm{C}$, causing the solder to reflow; during cooling the solder solidifies and bonds the two surfaces. Typically, it is recommended to reflow in a reduced oxygen environment such as a vacuum or nitrogen furnace, however, successful attachments have been performed in air using a hot plate. Finally, the device is wire-bonded in preparation for further processing.

\section{Glass Lid Fabrication with Getters}

As mentioned previously, plated parts can be a significant source of outgassing, which can cause a dramatic degradation in pressure due to the relatively small cavity volumes used in MEMS packages. While bake outs can accelerate desorption, any remaining trapped gas in the materials must be removed in order to maintain the sealed vacuum level. It is generally accepted that using getter material is the most reliable solution for maintaining and stabilizing pressures in hermetically sealed cavities [5].

In order to incorporate getters into the presented packaging procedure, an approach using custom fabricated glass lids was developed. The process starts with a four inch D263 glass wafer (purchased from Mark Optics) which was chosen because its coefficient of thermal expansion (CTE) is close to the CTE of the alumina package. Metalized sealing rings corresponding to the cavity size of the 24 pin ceramic DIP are defined on the wafer via lift-off using $500 \AA$ chromium and $5000 \AA$ of gold. Next, the wafer is sent to SAES Getters for the thin film deposition of their PageWafer getter, which is specifically designed to remove the most common gases present in MEMS cavities. A photograph of the glass wafer after getter deposition is presented in Figure 2. Finally, individual glass lids are obtained by dicing between the sealing rings; since the getter has a passivation layer to allow handling in atmospheric conditions, the use of clean water during dicing does not affect its performance. 


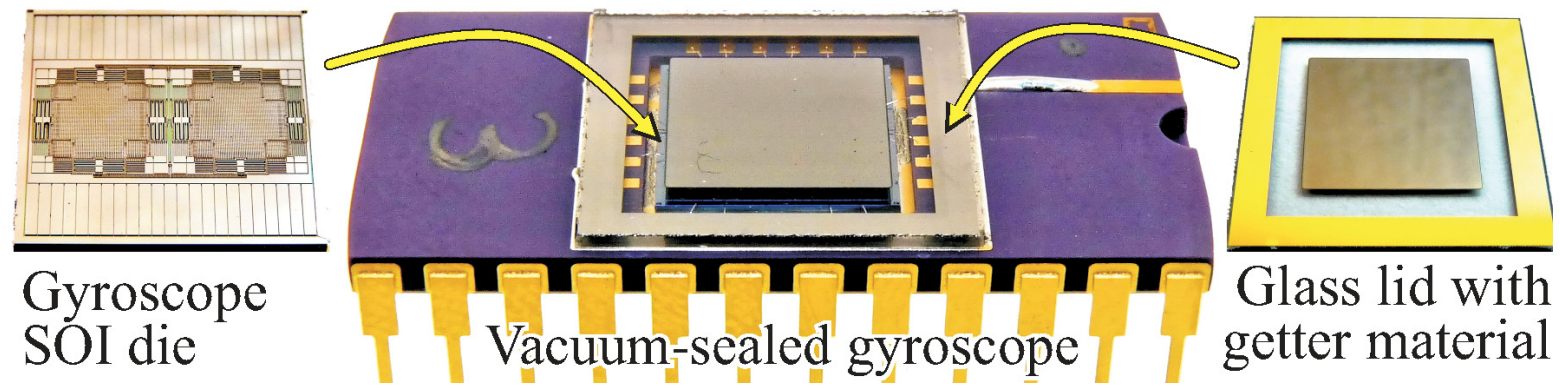

Figure 3. Vacuum packaged gyroscope prototype sealed using gold-tin solder and custom glass lid with getters.

\begin{abstract}
Ultra-High Vacuum Sealing and Wire-Bonding
Once the die attachment has been completed and the custom glass lids are fabricated and diced, the next step is the hermetic sealing of the cavity in an ultrahigh vacuum environment. Similar to the die attachment process, preform frames of $80 / 20$ gold-tin eutectc solder are used as the bonding material. Since the getter requires an elevated temperature activation to remove the passivation layer, the lids cannot have the solder pre-attached. Therefore, the preform must be attached to the sealing ring of the ceramic package. Since the package is suspended upside down during the process, the preform must be tacked to the sealing ring without reflowing it; this attachment can be done by hand using a hot soldering iron or done automatically using a programmable tack-welder.
\end{abstract}

Gold-tin solder can be used for both sealing steps because the melting temperature near the $80 / 20$ eutectic point undergoes a steep change versus weight percent. If the composition is changed to $18 \%$ tin for instance, the melting temperature becomes $350{ }^{\circ} \mathrm{C}$, while $16 \%$ tin melts at $400{ }^{\circ} \mathrm{C}$ [7]. As the diffusion of gold from the package and the die is inevitable during the reflow process, the composition is changed ultimately raising the melting temperature of the reflowed solder. Therefore, subsequent reflow process generally will not adversely affect the previous bonds.

The vacuum sealing process for this work was performed by SST International using their Model 3150 Programmable High Vacuum Furnace which is capable of pumping down to $10^{-6}$ Torr pressures and below. Both the lid and package are placed in high vacuum and baked out to accelerate outgassing from the plated metals. Next, the getter film is activated by heating the lid, exposing the gas absorbing material to the environment. To prevent premature solder reflow, the package is thermally shielded during the activation. The ceramic package is interfaced with the lid and weights are applied to the package, applying pressure during the reflow process to ensure a void-free bond. Finally, the cavity is sealed by heating the sys-

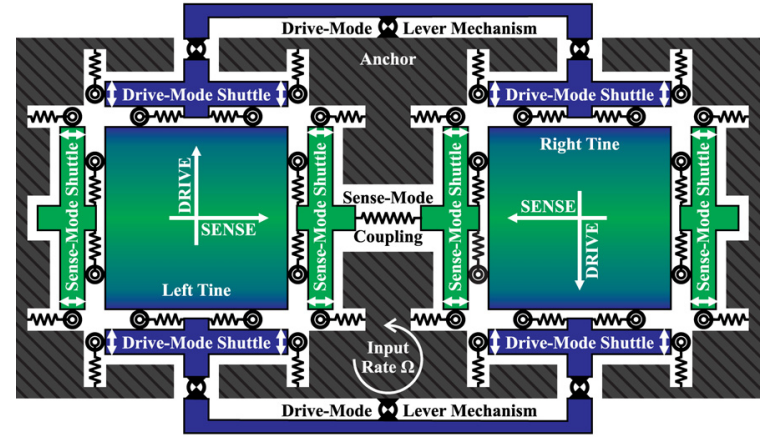

Figure 4. Structural layout of anti-phase operated gyroscope with sense-mode quality factor optimization.

tem above the $278{ }^{\circ} \mathrm{C}$ eutectic temperature causing the preform to reflow, bonding the two surfaces.

The result of the ultra-high vacuum sealing process is pictured in Figure 3, which shows a completely packaged SOI gyroscope [4] using a custom fabricated glass lid with getter material. As can be seen, a solder fillet is visible at the interface between the lid and the ceramic package indicating that a successful bond was achieved. In order to more completely verify the lid sealing process, the packages were inspected using $\mathrm{x}$ rays which revealed void free bonds for both the lid and die.

\section{EXPERIMENTAL DEMONSTRATION}

In order to demonstrate the feasibility of realizing ultra-high vacuum levels in sealed package cavities, a tuning fork gyroscope prototype [4] was packaged using the described procedure. The design, presented in Figure 4, is optimized to minimize the dissipation of energy through the substrate while prioritizing the sense mode quality factor. Using a sub-mTorr vacuum chamber, drive and sense mode quality factors of 67,000 and 125,000, respectively, were obtained for a $2.5 \mathrm{kHz}$ frequency indicating its potential for ultrahigh vacuum packaging. 


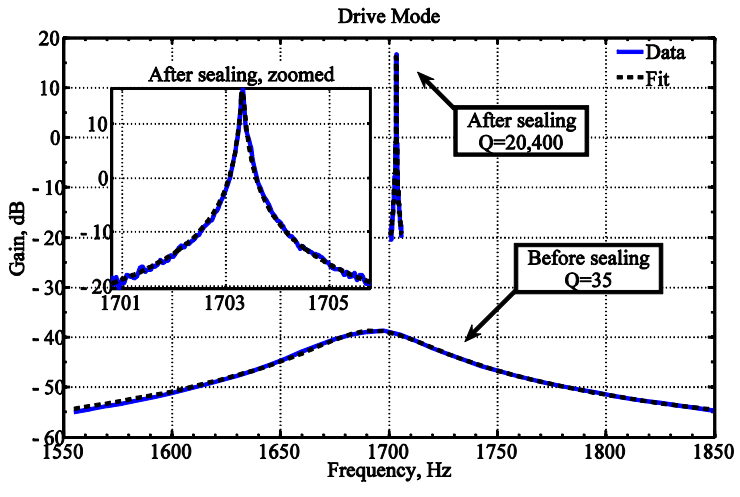

Figure 5. Drive mode before and after sealing.

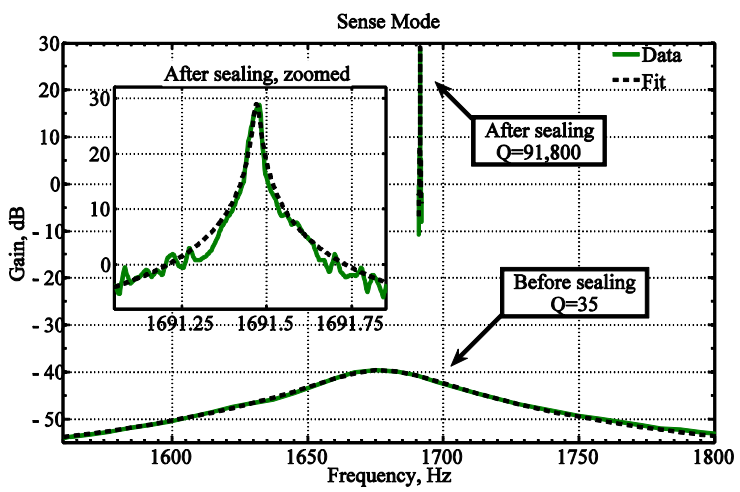

Figure 6. Sense mode before and after sealing.

The completely vacuum packaged gyroscope pictured in Figure 3 was characterized to determine the ultimate sealed cavity pressure. Figures 5 and 6 present the experimentally measured frequency response curves of the drive and sense modes, respectively, before and after vacuum sealing. The combination of the anti-phase design and versatile packaging process resulted in stand-alone quality factors of 20,400 and 91,800 at $1.7 \mathrm{kHz}$ thereby confirming the packaging approach for achieving sub-mTorr sealed pressures. To verify the stability of the vacuum level, the sense mode was characterized again four months after sealing. Figure 7 presents the experimental frequency response curve revealing a stable Q of $92,600 \pm 2 \%$.

\section{CONCLUSIONS}

A flexible vacuum sealing approach using commercially available materials and processes was developed and demonstrated to provide sub-mTorr pressures in sealed cavities. Using optimized, high-Q mechanical designs, stand-alone quality factors on the order of 100,000 were successfully achieved. While the described process is currently intended for package level sealing, it also enables a path to wafer-level vacuum packaging using the glass as a capping layer.

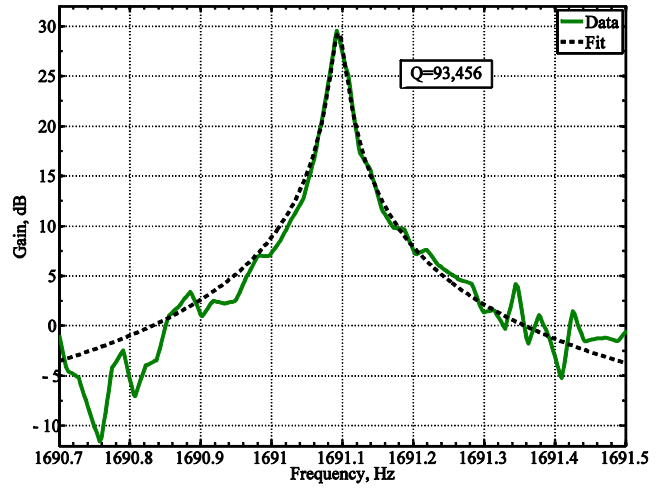

Figure 7. Sense mode four months after sealing.

\section{ACKNOWLEDGEMENTS}

This work was supported by the Office of Naval Research/Naval Surface Warfare Center contract N00178-08-C1014. The authors would like to thank Heather Florence of SAES Getters and Paul Barnes of SST International for his guidance during the vacuum sealing and graphite parts manufacturing. The gyroscopes were designed and characterized at the MicroSystems Laboratory, University of California, Irvine.

\section{REFERENCES}

[1] K. Najafi, "Micropackaging Technologies for Integrated Microsystems: Applications to MEMS and MOEMS," Proc. of SPIE, vol. 4979, pp. 119, 2003.

[2] B. Kim, M. Hopcroft, C.M. Jha, et al., "Using MEMS to Build the Device and Package," Proc. of Transducers 2007, pp. 331-334, Lyon, France, June 10-14, 2007.

[3] S.-H. Lee, J. Cho, S.W. Lee, et al., "A LowPower Oven-Controlled Vacuum Package Technology for High-Performance MEMS," Proc. of IEEE MEMS 2009, pp. 753-756, Sorrento, Italy, January 25-29, 2009.

[4] A.A. Trusov, A.R. Schofield, and A.M. Shkel, "Gyroscope Architecture with Structurally Forced Anti-Phase Drive-Mode and Linearly Coupled Anti-Phase Sense-Mode," Proc. of Transducers 2009, pp. 660-663, Denver, CO, June 21-25, 2009

[5] R. Ramesham and R. C. Kullberg, "Review of vacuum packaging and maintenance of MEMS and the use of getters therein," J. Micro/Nanolith. MEMS MOEMS, vol. 8, pp. 031307, July 2009.

[6] A. A. Trusov, A. R. Schofield and A. M. Shkel, "A substrate energy dissipation mechanism in inphase and anti-phase micromachined z-axis vibratory gyroscopes," J. Micromechanics and Microengineering, vol. 18, pp. 095016, 2008

[7] Indium Corporation, "Gold Tin - The Unique Eutectic Solder Alloy,” Application Note. 